\title{
Effect of parametric uncertainties on vibration mitigation with periodically distributed and interconnected piezoelectric patches
}

\author{
Journal Title \\ $\mathrm{XX}(\mathrm{X}): 1-12$ \\ (C)The Author(s) 2020 \\ Reprints and permission: \\ sagepub.co.uk/journalsPermissions.nav \\ DOI: $10.1177 /$ ToBeAssigned \\ www.sagepub.com/ \\ SAGE
}

\author{
Marcelo A. Trindade ${ }^{1}$, Boris Lossouarn ${ }^{2}$ and Jean-François Deü ${ }^{2}$
}

\begin{abstract}
This work presents an analysis of the effect of parametric uncertainties on the vibration control performance of a rod with periodically distributed piezoelectric patches that can be either independently connected to electrical shunt circuits or interconnected through an electrical line of inductors. In both cases, the capacitance of the piezoelectric patches is considered as stochastic parameters following a known probability density function distribution. Then, Monte Carlo simulations are performed to evaluate mean values and confidence intervals of the frequency response functions to assess the robustness of each solution and to compare different solutions in terms of nominal and robust performances. Results have shown that vibration amplitude reduction worsen significantly due to the mistuning between structural natural frequency and circuit resonance frequency. Yet, interconnected circuits are more robust to these uncertainties than independent shunts because they ensure a mean response that is closer to the nominal one. It was then proposed to assess the effect of modifying the circuits' resistance. Results have shown that increased resistance decreases variability when considering both environmental and manufacturing variabilities. This also favors the use of interconnected circuits that require increased resistance for robust vibration mitigation.
\end{abstract}

\section{Keywords}

uncertainty quantification, piezoelectric materials, vibration control, shunted damping

\section{Introduction}

Piezoelectric materials have been widely used as distributed sensors and actuators to control structural vibrations of flexible structures. They are normally bonded to a host structure and connected to an electrical circuit. Therefore, they provide an electromechanical coupling to the host structure that, depending on electrical connections, can be used for several purposes such as passive, semi-passive, active or active-passive vibration control (Hagood and von Flotow 1991; Ahmadian and DeGuilio 2001). When distributed periodically, it was shown that they can also be used to provide stop bands for wave propagation applications (Thorp, Ruzzene and Baz 2001, 2005), to design acoustic metamaterials (Airoldi and Ruzzene 2011; Celli and Gonella

2015) or to create electromechanical phononic crystals (Bergamini et al. 2015; Flores Parra et al. 2017).

In the case of passive vibration control applications, most studies focused on shunt circuits that connect the two electrodes of each piezoelectric patch. The most common circuits are resistive and resonant (resistive-inductive) ones but several research works proposed more sophisticated circuits using, for instance, switch or negative capacitance circuits (Hollkamp 1994; Reza Moheimani 2003). More recently, Maurini, Dell'Isola and Del Vescovo (2004) and Lossouarn, Aucejo and Deü (2015) considered the interconnection of a network of piezoelectric patches through resonant electrical circuits in a way that good multimodal vibration attenuation performance can be obtained with much smaller inductance values, compared to resonant shunt circuits.

However, as for the shunted case, the vibration control performance is dependent on the adequate tuning between electrical circuit components and structure's mechanical properties (Valis, von Flotow and Hagood 1994). Thus, variabilities and/or uncertainties on material properties, boundary conditions, and environmental conditions can have a major effect on reducing the expected or predicted performance of such devices. Nevertheless, few studies attempted to analyze the effect of parametric uncertainties on passive vibration control using shunted piezoelectric patches (Andreaus and Porfiri 2007; Santos and Trindade 2011; Berardengo et al. 2015) and none considering interconnected networks.

This paper begins with the description of the considered problem involving a rod covered with an array of piezoelectric patches, whose electrodes are connected to

\footnotetext{
${ }^{1}$ Department of Mechanical Engineering, São Carlos School of Engineering, University of São Paulo, Av. Trabalhador São Carlense, 400, São Carlos, SP, 13566-590, Brazil

${ }^{2}$ Laboratoire de Mécanique des Structures et des Systèmes Couplés (LMSSC), Conservatoire national des arts et métiers (Cnam), 292 Rue Saint-Martin, 75003 Paris, France
}

\section{Corresponding author:}

Marcelo A. Trindade, Department of Mechanical Engineering, São Carlos School of Engineering, University of São Paulo, Av. Trabalhador São Carlense, 400, São Carlos, SP, 13566-590, Brazil.

Email: trindade@sc.usp.br 
an electrical network made of inductors and resistors. A second section describes the methodology used in this study, where the objective is to evaluate the effect of parametric uncertainties at local or global levels. The results are then analyzed in the third section that shows the variability induced in the frequency response functions. A fourth section investigates the effect of the circuits' resistance on the robustness of vibration mitigation solutions. This leads to potential conclusions regarding the choice of optimal independent shunts or interconnected piezoelectric networks in the fifth section. Finally, a last section is presented with some perspectives on the effect of uncertainties in multimodal vibration mitigation using interconnected circuits.

\section{Problem description}

A free-free AU4G duraluminum rod in longitudinal vibration with length $l_{s}=1000 \mathrm{~mm}$, width $b=20 \mathrm{~mm}$ and thickness $h_{s}=20 \mathrm{~mm}$ is considered. The rod has mass density $\rho_{s}=$ $2780 \mathrm{~kg} \mathrm{~m}^{-3}$ and Young modulus $Y_{s}=73.9 \mathrm{GPa}$. A loss factor of $0.5 \%$ is considered for the rod. 20 pairs of PIC151 piezoelectric patches are bonded to the rod following the layout in Figure 1 which only shows 10 unit cells. Each patch has the following dimensions: length $l_{p}=30 \mathrm{~mm}$, width $b=20 \mathrm{~mm}$ and thickness $h_{p}=0.5 \mathrm{~mm}$. The piezoelectric patches are considered to be equal and all have mass density $\rho_{p}=7800 \mathrm{~kg} \mathrm{~m}^{-3}$, Young modulus at zero electric field $Y_{p}^{E}=66.7 \mathrm{GPa}$, piezoelectric charge constant $d_{31}=$ $-210 \mathrm{pC} \mathrm{N}^{-1}$ and dielectric permittivity $\epsilon_{33}^{\sigma}=21.2 \mathrm{nF} \mathrm{m}^{-1}$.

For the modeling of the rod with periodically bonded piezoelectric patches, the transfer matrix approach was used. For that, 20 electromechanical unit cells that periodically repeat all along the structure were considered, following the layout in Figure 1 which only shows 10 unit cells. The unit cell is composed of a portion of the rod with length $a=50 \mathrm{~mm}$, two entire piezoelectric patches bonded onto the upper and lower surfaces of the rod, centered along the length and poled in opposite through-thickness directions, one (independent) shunt circuit that connects directly the electrodes of these patches and two circuits that connect the pair of patches to left and right neighboring unit cells circuits.

The obtained model using the transfer matrix approach is presented in detail in Lossouarn, Aucejo and Deü (2015) and is not repeated here for the sake of brevity. The model is based on a homogenized electromechanical unit cell with mechanical and electrical degrees of freedom that is able to account for both shunted and interconnected circuits cases. It is assumed that the rod is excited by a point longitudinal force applied at its left end and that the longitudinal velocity at the right end of the rod is measured. Then, the vibration control performance is assessed using the mobility frequency response function around the first vibration mode of the rod.

\section{Methodology}

In order to evaluate the effect of parametric uncertainties in the performance of independent resonant shunt circuits and interconnected circuits for vibration mitigation, the two cases were analyzed separately. To obtain only the first case (shunted patches), the resistance of the interconnected
Table 1. Inductance and resistance values for the independent and interconnected circuits used for the nominal model.

\begin{tabular}{lcc}
\hline & Independent circuit & Interconnected circuit \\
\hline Inductance $(\mathrm{mH})$ & 113.5 & 2.795 \\
Resistance $(\Omega)$ & 142.3 & 3.503 \\
\hline
\end{tabular}

circuits $\left(R_{s}\right)$ is set to a very large value to represent opening these interconnection circuits. Contrarily, to obtain the second case (interconnected patches), the resistance of the shunt circuits $\left(R_{s h}\right)$ is set to a very large value to represent opening the shunt circuits. The use of both independent and interconnected circuits simultaneously was not considered.

Then, the vibration mitigation performance, focusing mainly on the first vibration mode, using the two solutions was evaluated and compared. First, the nominal case was defined using the optimal values for the circuit components. Then, the parameters to be considered uncertain were defined. For these, stochastic models were constructed and used for Monte Carlo simulations. Finally, the results are used to assess the robustness of the two solutions.

\section{Nominal case}

Initial values for the resistance and inductance of the independent and interconnected circuits were taken from (Lossouarn, Aucejo and Deü, 2015) and, then, fine-tuned using a Nelder-Mead simplex algorithm (fminsearch) to minimize the $H_{\infty}$ norm of the FRF (Table 1). Notice that for experimental implementation, inductance and resistance values can only approximate the optimal ones. As proposed by Lossouarn, Aucejo and Deü (2015), whenever measured inductance of actual inductors is smaller than expected, the tuning can be corrected by adding capacitors in parallel to the piezoelectric patches. In the case of the resistance, the optimal values also account for the internal resistance of the inductors. Whenever the optimal resistance is larger than the internal resistance of the inductors, resistors can be added to the circuit.

The nominal mobility frequency responses of the rod with independent and interconnected circuits, compared to the open circuit case (all circuits opened), are shown in Figure 2. It is noticeable that the independent circuits are only effective in reducing the vibration amplitude around the first resonance frequency, since all circuits (for all patches) were tuned to that frequency. On the other hand, the interconnected circuits are able to reduce the vibration amplitude over the entire frequency range considered, although they seem to be more effective around the first resonance frequency. This is because their nominal resistance was tuned aiming preferentially at the first mode. An increase in resistance could better reduce the peaks clearly observed for the second to fourth resonances, but at the cost of worsening the performance for the first mode. When considering only the response around the first mode, both independent and interconnected circuits lead to the same performance, with around $20 \mathrm{~dB}$ amplitude reduction when compared to the open circuit case. In the present work, the focus will be first put on the response around the first mode to allow a fair comparison between independent and interconnected circuits. 


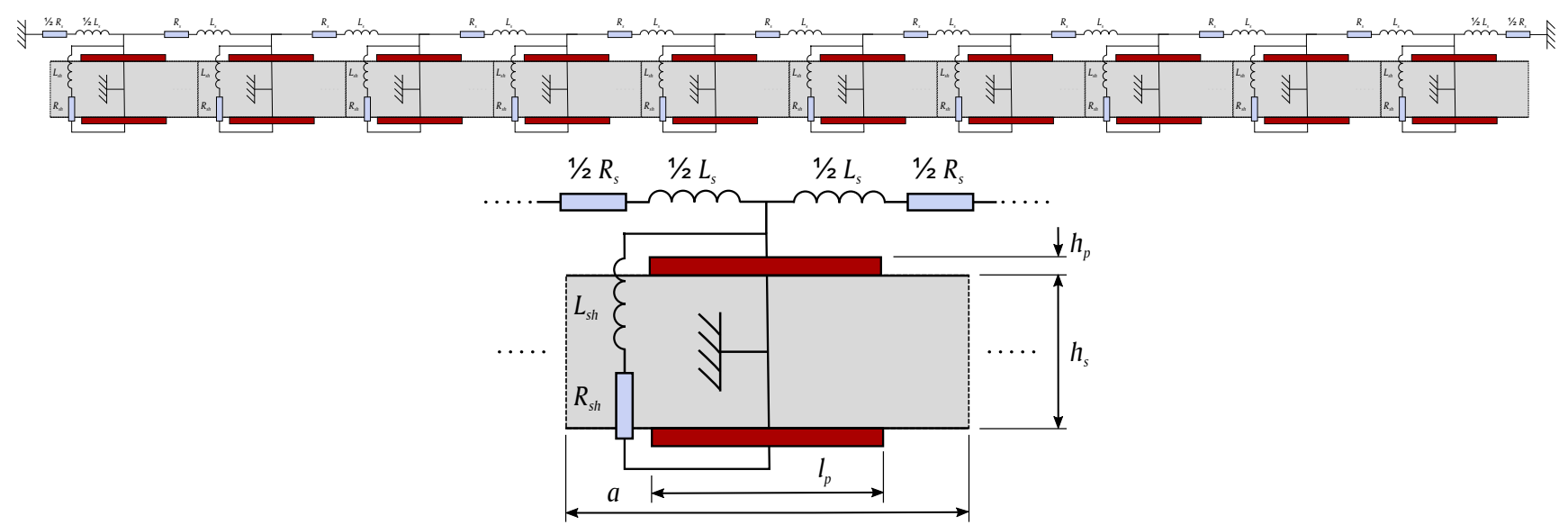

Figure 1. Example of a rod with 10 pairs of periodically bonded piezoelectric patches connected to independent $\left(L_{s h}\right.$ and $\left.R_{s h}\right)$ and interconnected $\left(L_{s}\right.$ and $\left.R_{s}\right)$ circuits with detailed unit cell.

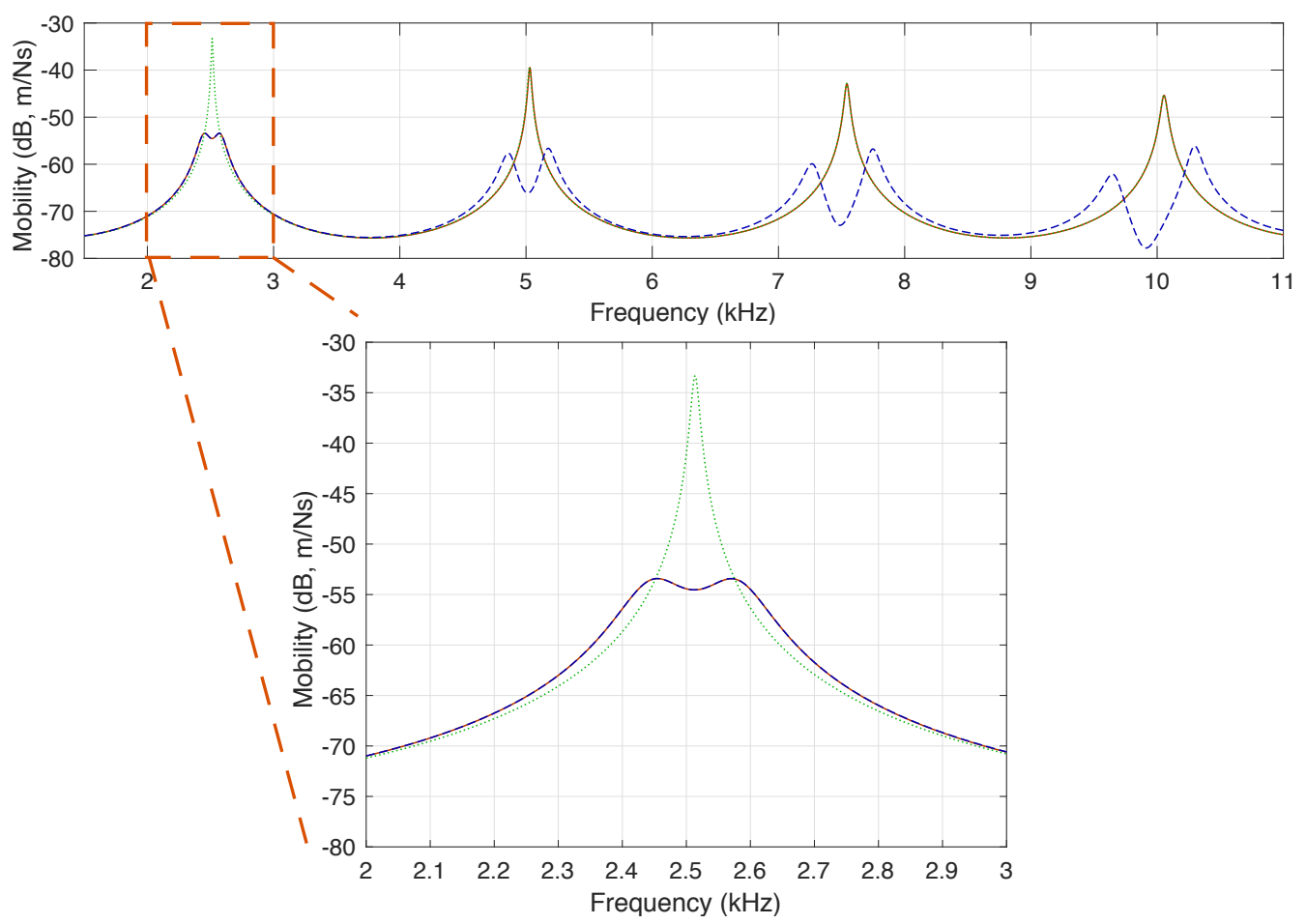

Figure 2. Nominal frequency response of the rod: open-circuit (dot), independent circuits (solid), interconnected circuits (dash).

The predicted vibration amplitude reduction performance is very much dependent on a proper tuning between structure's targeted natural frequency, piezoelectric coupling, piezoelectric effective capacitance and circuit's inductance and resistance. Thus, if any of these parameters are different from the nominal ones, one should expect to observe different actual performances. Figure 3 a first investigates the effect of capacitance variations on the $H_{\infty}$ norm of the mobility function. Once the inductance is fixed, capacitance variations have a strong influence as a $10 \%$ variation can increase by more than $150 \%$ the maximum value of the FRF. Moreover, inductance variations lead to similar observations. This confirms that classical results, obtained with a onedegree-of-freedom mechanical system (Preumont 2018), apply to both the interconnected and independent multishunt rods. The numerical analysis gives similar results for both circuits, which shows that they are equivalent around the first mode of the rod. Resistance variations are also investigated to confirm that they have a weaker influence on the results: around $30 \%$ increase of the $H_{\infty}$ norm for a $50 \%$ variation of the resistance, as seen in Figure $3 b$.

The considered nominal resistance is the one that minimizes the $H_{\infty}$ norm after optimal tuning of the capacitance and inductance values. Yet, it is possible to focus on another optimization method based on maximum damping. This corresponds to maximizing the minimum damping ratio of the considered electromechanical system that is equivalent to a two-degrees-of-freedom system over the frequency range of interest. To do so, one can extract the modal parameters of the FRF subjected to variation of the capacitance or resistance values. The two close modes of the tuned-mass-damper-type system are associated with two eigenfrequencies and two damping ratios. Figure 4a shows that the optimum in terms of maximum damping is obtained 

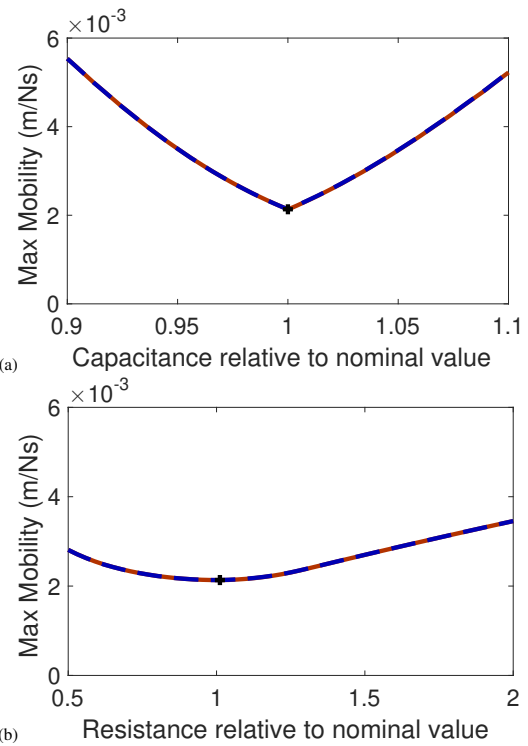

Figure 3. Effect of circuits' capacitance and resistance on the $H_{\infty}$ norm of the mobility function: independent circuits (solid), interconnected circuits (dash).
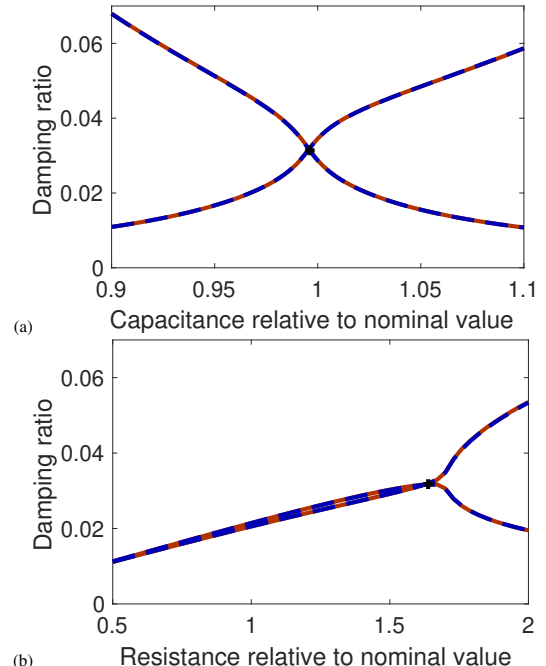

Figure 4. Effect of circuits' capacitance and resistance on the damping ratios: independent circuits (solid), interconnected circuits (dash)

with a capacitance value close to the one considered during the previous optimization. However, Figure $4 \mathrm{~b}$ leads to an optimal resistance about $\sqrt{8 / 3} \approx 1.63$ times the previous nominal value. This is fully consistent with results obtained with classical resonant shunts when the two loops of the root locus plot crosses at a single point of maximum damping (Preumont 2018).

\section{Uncertain parameters}

There are a number of factors that lead to variability of theoretical performance prediction. Here, it is assumed that the model used is accurate enough for the present analysis. However, the model parameters can be subjected to variability (uncertainty). The parameters that most affect the resonance frequency tuning are the piezoelectric effective capacitance and the circuits' inductance. Also, experimental tests have shown that even homemade inductances can have very small tolerances and can be adapted if needed (Darleux, Lossouarn and Deü 2018). On the other hand, the capacitance of piezoelectric patches is more difficult to control and could present significant variability when subjected, for instance, to temperature variations. They also present variability due to manufacturing tolerances (in both material properties, such as the dielectric coefficient, and geometrical properties, electrode area and patch thickness). For these reasons, in the present work, the piezoelectric patches capacitances will be considered as subjected to variability.

In a tentative to separate two potential sources of uncertainty of capacitance values, one related to the variability of the patches themselves (e.g. material and geometrical properties) and one related to environmental conditions that could affect all the patches (e.g. temperature), two multiplicative stochastic parameters were defined, $f_{i}$ and $f_{g}$. One $\left(f_{g}\right)$ that varies the capacitance of all patches simultaneously and another $\left(f_{i}\right)$ that varies the capacitance of each patch relative to the global value. Their effects can be combined to generate the overall capacitance of each patch, such that the capacitance of the $i$-th patch would be

$$
\hat{C}_{p i}=\bar{C}_{p} \hat{f}_{g} \hat{f}_{i}
$$

where $\bar{C}_{p}$ is the nominal (expected) value for the patch capacitance, $\hat{f}_{g}$ is a stochastic variable related to the environmental (global) variability and $\hat{f}_{i}$ is a stochastic variable related to the manufacturing (individual) variability. Notice that only $\hat{f}_{i}$ may be different from one patch to another.

\section{Stochastic modeling}

The probability density function (p.d.f.) for the stochastic variables are not known a priori, although it is expected that a mean (or nominal) value could be known and, also, that the variables should be positive. The Maximum Entropy Principle (Jaynes 1957; Soize 2001) states that one should consider a probability distribution that maximizes the uncertainties using only the available information on the random variable. It also guarantees that mathematical and statistical properties of the random variable are respected.

In the present case, the capacitance has to be a positivevalued random variable, which is a mathematical property that defines the support for the p.d.f. as $] 0,+\infty[$. It also has to be a second-order random variable, such that the expectation of the squared variable is finite, $E\left\{X^{2}\right\}<$ $+\infty$. This translates into a p.d.f. for which the probability diminishes as the variable tends to infinity. The inverse of the capacitance is also used for the problem solution and, thus, it has to be also a second-order random variable, such that the expectation of the inverse squared variable is also finite, $E\left\{X^{-2}\right\}<+\infty$. This translates into a p.d.f. for the which the probability diminishes as the variable tends to zero. Finally, the mean value $E\{X\}=\bar{X}$ is considered to be known and equal to be the nominal value.

Using this information, the Maximum Entropy Principle leads to a Gamma probability density function, such that

$$
p_{X}(X)=\mathbb{I}_{] 0,+\infty[}\left(\frac{1}{\delta_{X}^{2} \bar{X}}\right)^{\delta_{X}^{-2}} \frac{X^{\delta_{X}^{-2}-1}}{\Gamma\left(\delta_{X}^{-2}\right)} \exp \left(-\frac{X}{\delta_{X}^{2} \bar{X}}\right)
$$


in which $\delta_{X}=\sigma_{X} / \bar{X}$ is the relative dispersion of the stochastic parameter and $\sigma_{X}$ is its standard deviation. The Gamma function is defined as $\Gamma(x)=\int_{0}^{\infty} t^{x-1} e^{-t} \mathrm{~d} t$. In the present case, $X$ assumes the multiplicative parameters $f_{i}$ and $f_{g}$. The deterministic optimal value for the capacitance is assumed as its nominal and mean values. Thus, the nominal values for both $\hat{f}_{g}$ and $\hat{f}_{i}$ are considered to be unitary. The relative dispersion for both manufacturing and environmental causes is actually unknown a priori. Previous observation (Darleux, Lossouarn and Deü 2018) has shown that capacitance values can vary up to $12 \%$ for a $38^{\circ} \mathrm{C}$ temperature variation. However, since there might be several different sources for both manufacturing and environmental uncertainties, the actual relative dispersions should be estimated for a given application condition. Therefore, in the present case, a parametric analysis will be carried out considering two arbitrary, but representative, values for the relative dispersion, $5 \%$ and $10 \%$, to better understand their effect on the vibration mitigation performance.
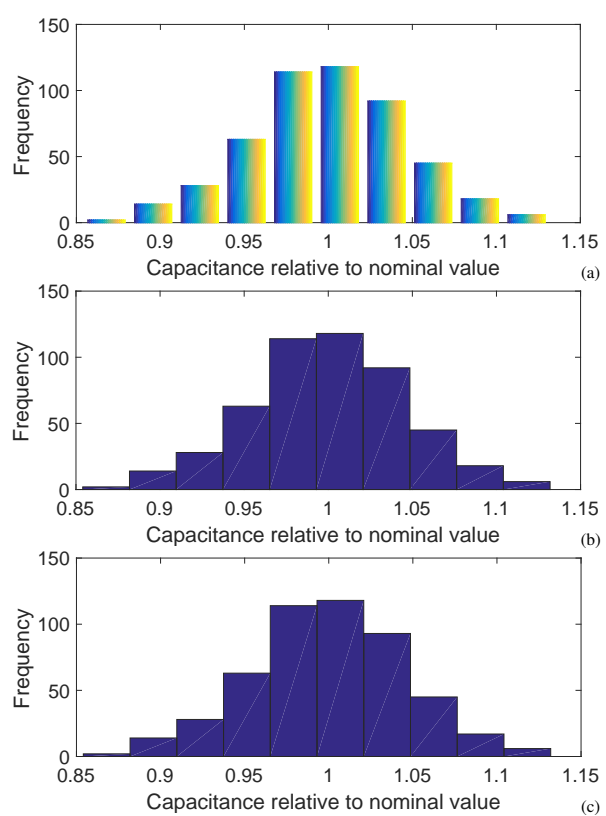

Figure 5. Histogram of capacitance with $5 \%$ global relative dispersion: (a) all patches, (b) first patch, (c) second patch.

Figures 5 and 6 show histograms for the relative capacitances (relative to the nominal value). In Figure 5, a $5 \%$ relative dispersion is considered only for environmental uncertainties (that is, $f_{g}$ has $5 \%$ relative dispersion and $f_{i}$ is unitary and constant). On the other hand, in Figure 6, a 5\% relative dispersion is considered for both manufacturing and environmental uncertainties (that is, both $f_{i}$ and $f_{g}$ have $5 \%$ relative dispersions). In both figures, the histograms of the capacitances of the two first patches are also shown. Notice that, in the second case (Figure 6), the capacitance values of first and second patches may be different, although following the same probability density function. This does not happen for the first case (Figure 5), which involves no individual variability.

\section{Monte Carlo simulations}

Considering the stochastic model presented previously for the two parameters affecting the piezoelectric patch
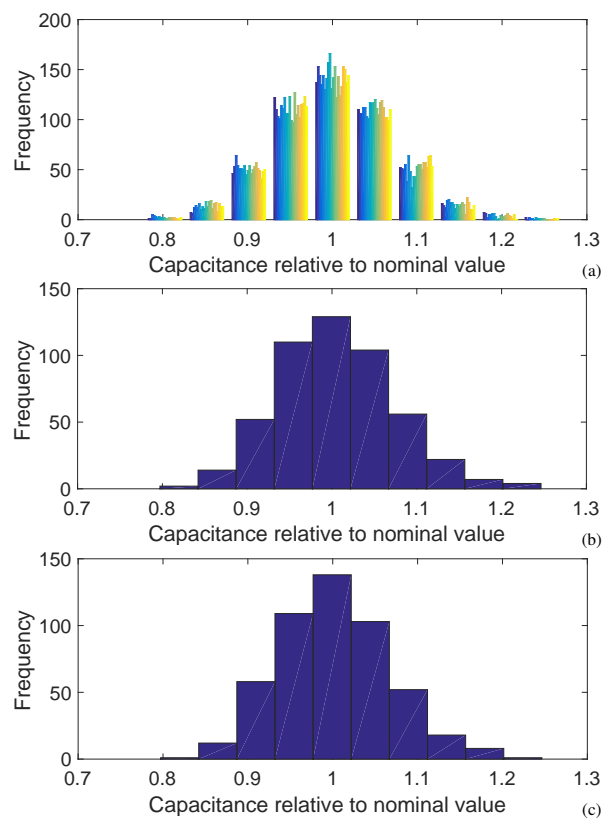

Figure 6. Histogram of capacitance with $5 \%$ individual and global relative dispersions: (a) all patches, (b) first patch, (c) second patch.

capacitance, $f_{i}$ and $f_{g}$, random realizations of the corresponding stochastic variables, $\hat{f}_{i}$ and $\hat{f}_{g}$, were then generated using MATLAB function gamrnd. In all cases, two values for the relative dispersions were considered, $5 \%$ and $10 \%$. Some combinations of parametric uncertainties were performed for the two parameters considered.

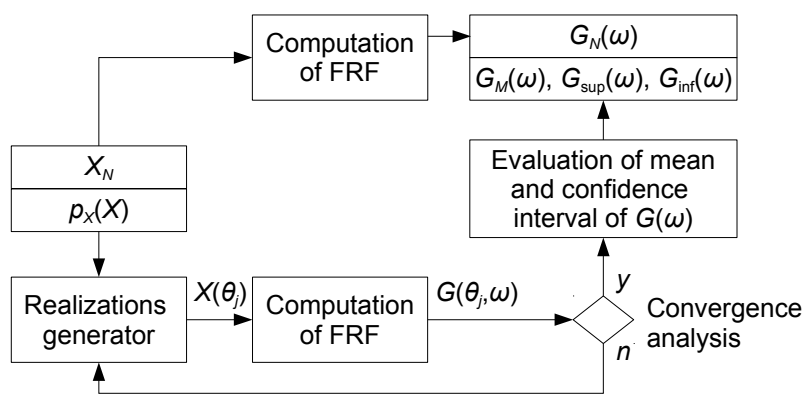

Figure 7. Schematic representation of Monte Carlo simulations.

Then, Monte Carlo simulations were performed for the studied cases by varying the relative dispersions and sample size. The procedure is schematically shown in Figure 7. The first step is to generate $N$ realizations (samples), $\theta_{j}$, of the stochastic variables, $\hat{f}_{i}\left(\theta_{j}\right)$ and $\hat{f}_{g}\left(\theta_{j}\right)$, using the Gamma probability density function presented previously. The mobility frequency response function, $G\left(\theta_{j}, \omega_{k}\right)$ with $j=1, \ldots, N$, is evaluated for each set of parameters, which include the realizations of the stochastic variables, $\hat{f}_{i}\left(\theta_{j}\right)$ and $\hat{f}_{g}\left(\theta_{j}\right)$, using the same model and procedure considered for the nominal case. All other material and geometrical properties of the rod and patches as well as the circuits' resistance and inductance are kept unchanged. Thus, only the patches' capacitances are varied from one analysis to another. 
Next, in order to quantify the uncertainty of the frequency response due to the uncertainty of the patches' capacitances (or how the parametric uncertainty propagates to the frequency response), the $N$ realizations of the frequency response, $G\left(\theta_{j}, \omega_{k}\right)$ with $j=1, \ldots, N$, at any given frequency point $\omega_{k}$, were used to evaluate their mean values $G_{M}\left(\omega_{k}\right)$ and their $2.5 \%$ and $97.5 \%$ percentiles, $G_{i n f}\left(\omega_{k}\right)$ and $G_{\text {sup }}\left(\omega_{k}\right)$, respectively.

The maximum, $G_{\text {sup }}\left(\omega_{k}\right)$, and minimum, $G_{\text {inf }}\left(\omega_{k}\right)$, values for all frequency points define curves that represent, respectively, the upper and lower bounds of the $95 \%$ confidence interval. This means that, provided the assumed stochastic model is satisfactory, the actual frequency response curve, for a given value of the capacitance, must be inside the confidence interval. Notice that the mean FRF curve does not necessarily represent an attainable actual response but, assuming a Gaussian-like posterior probability distribution, most actual FRF curves should be closer to the mean FRF curve than to the lower or upper bounds. The nominal FRF, $G_{N}\left(\omega_{k}\right)$, is obtained considering the nominal values for the stochastic variables (capacitance).

Some preliminary convergence tests were carried out to determine a reasonable sample size for the Monte Carlo simulations. For that, the Euclidean norm of the mobility frequency response function, around the first resonance frequency $([2000,3000] \mathrm{Hz})$, was evaluated for each realization of the stochastic parameters considering simultaneously $5 \%$ relative dispersion for $f_{i}$ and $10 \%$ relative dispersion for $f_{g}$. Figure 8 shows the normalized response versus the Monte Carlo simulations performed. It indicates that $N=500$ samples seem to be enough even for a case with high dispersions.

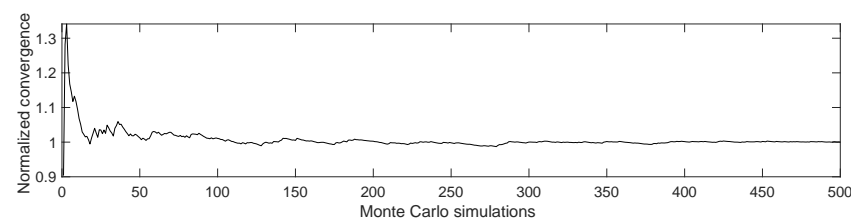

Figure 8. Convergence of Monte Carlo simulation for the norm of the frequency response of a rod with independent circuits for uncertain capacitance (5\% individual plus $10 \%$ global dispersion).

\section{Uncertainty quantification results}

In this section, the effects of global (e.g. environmental) and individual (e.g. manufacturing) uncertainties of the piezoelectric patches' capacitance values on the frequency response of the rod, and ultimately on the vibration amplitude, are analyzed. The analyses are performed for both independent and interconnected circuits.

First, the cases were capacitance variabilities are mainly due to environmental conditions that could affect the ensemble of patches (e.g. temperature) are analyzed. For that, $f_{i}$ is assumed to be unitary and constant (deterministic), while $f_{g}$ assumes different values according to the stochastic model presented previously. Thus, $\delta f_{i}=0$ and $\delta f_{g} \neq 0$. Then, the frequency responses of the rod with independent and interconnected circuits were evaluated for two values
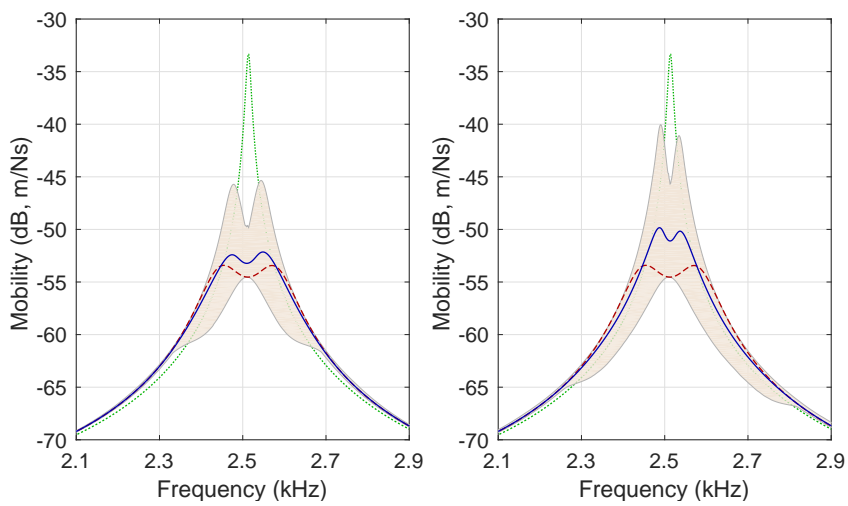

Figure 9. Frequency response of rod with independent circuits for uncertain patch capacitance (5\% (left) and 10\% (right) global dispersion): open-circuit (short dash), RL nominal (dash), RL mean (solid), 95\% confidence interval (fill).
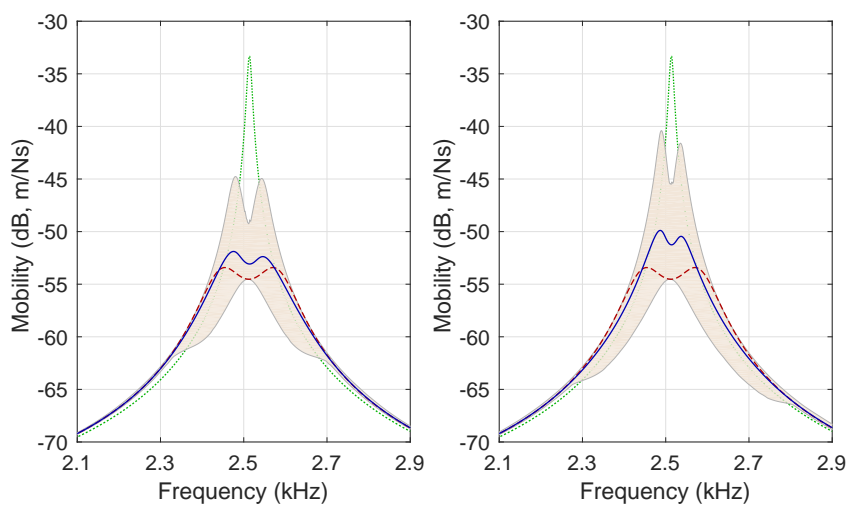

Figure 10. Frequency response of rod with interconnected circuits for uncertain patch capacitance (5\% (left) and 10\% (right) global dispersion): open-circuit (short dash), RL nominal (dash), RL mean (solid), 95\% confidence interval (fill).

of relative dispersion $\delta f_{g}, 5 \%$ and $10 \%$, and are shown in Figures 9 and 10.

In this case, it is possible to observe that the frequency responses for both independent and interconnected circuits are very close, from mean values and confidence intervals perspectives. In terms of the maximum responses, for the independent circuit, the difference between the maximum values of the nominal frequency response and the superior of the $95 \%$ confidence interval is $8.1 \mathrm{~dB}$ and $13.4 \mathrm{~dB}$ for $\delta f_{g}=$ $5 \%$ and $\delta f_{g}=10 \%$, respectively. For the interconnected circuit, these values are $8.7 \mathrm{~dB}$ and $13.0 \mathrm{~dB}$ for $\delta f_{g}=$ $5 \%$ and $\delta f_{g}=10 \%$, respectively. Therefore, these results suggest that, under global capacitance variabilities, the performance of independent and interconnected circuits are equivalent.

Next, only the capacitance variabilities due to patches individual properties are considered. For that, $f_{g}$ is assumed to be unitary and constant (deterministic), while $f_{i}$ may assume different values according to the stochastic model presented previously. Thus, $\delta f_{i} \neq 0$ and $\delta f_{g}=0$. Then, the frequency responses of the rod with independent and interconnected circuits were evaluated for the same values of relative dispersion $\delta f_{i}, 5 \%$ and $10 \%$. They are shown in Figures 11 and 12 .

It is noticeable that, for the interconnected circuit, the mean response and the superior of the confidence interval 

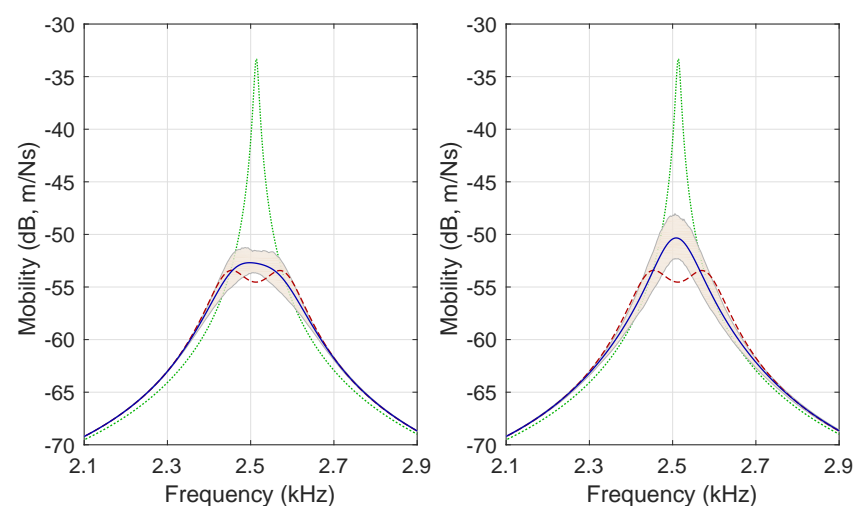

Figure 11. Frequency response of rod with independent circuits for uncertain patch capacitance (5\% (left) and 10\% (right) individual dispersion): open-circuit (short dash), $\mathrm{RL}$ nominal (dash), RL mean (solid), 95\% confidence interval (fill).
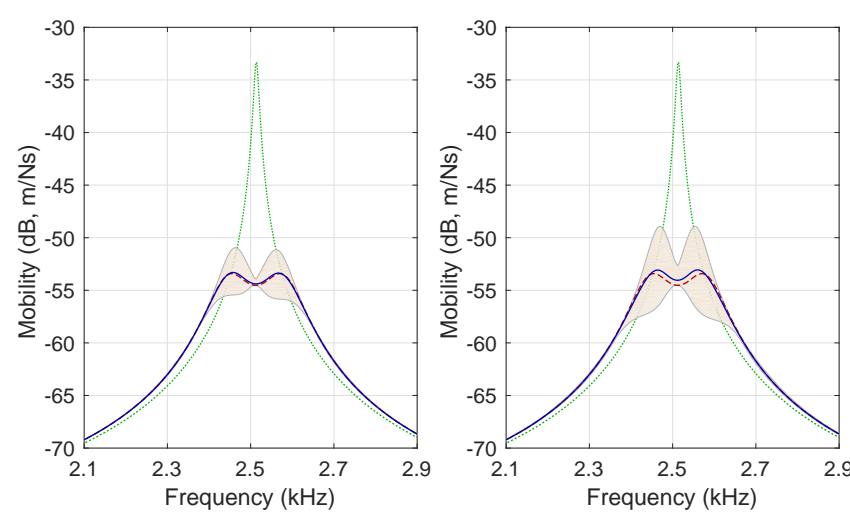

Figure 12. Frequency response of rod with interconnected circuits for uncertain patch capacitance (5\% (left) and 10\% (right) individual dispersion): open-circuit (short dash), RL nominal (dash), RL mean (solid), 95\% confidence interval (fill).

follows the shape of the nominal response with two peaks, while this is not the case for the independent circuit. In terms of the maximum responses, for the independent circuit, the difference between the maximum values of the nominal frequency response and the superior of the $95 \%$ confidence interval is $2.2 \mathrm{~dB}$ and $5.4 \mathrm{~dB}$ for $\delta f_{i}=5 \%$ and $\delta f_{i}=10 \%$, respectively. For the interconnected circuit, these values are $2.5 \mathrm{~dB}$ and $4.5 \mathrm{~dB}$ for $\delta f_{i}=5 \%$ and $\delta f_{i}=$ $10 \%$, respectively. These results suggest that, for the case of capacitance individual variabilities, the interconnected circuit is slightly more robust for large individual dispersion. But the main thing is that the nominal performance can be achieved with the interconnected circuit, while it cannot with the independent shunts (with a $95 \%$ confidence interval). The independent circuit leads to a mean FRF that is similar to the result of a resonant shunt with a resistance above its nominal value. This suggests that the circuits' resistance have a significant influence on the comparison between the robust performance of the interconnected and independent circuits.

\section{Effect of circuits' resistance on FRF variability}

Since the vibration amplitude reduction performance seems to be significantly affected by the patches' capacitance uncertainties, it is worthwhile to seek for solutions to mitigate the performance uncertainties. Previous results have shown that most of the uncertainty in the frequency response is due to the mistuning between structural natural frequency and circuit resonance frequency. That is because mistuning leads to the increase of one peak accompanied by the reduction of the other one (Santos and Trindade 2011). Therefore, it is expected that an increase of the circuits' resistance would reduce the increasing peak in mistuned cases and, thus, increase overall robustness to the considered parametric (capacitance) uncertainties.

Therefore, additional simulations were performed to evaluate the effect of increasing and reducing the circuits' resistance values. To assess the effect of circuit resistance on the nominal and robust vibration control performances of independent and interconnected circuits, the circuit resistance was varied from $-50 \%$ to $+100 \%$, with $10 \%$ steps, of its nominal value. The frequency responses of the rod with independent and interconnected circuits were then evaluated considering either individual or global capacitance variabilities and are shown in Figures 13, 14, 15 and 16.

It is noticeable that, in the case of individual variability in the capacitances and for decreased resistance values, the independent circuit leads to a mean response that is much smoother than the nominal one (Figure 13), whereas, for the interconnected circuit, the mean and nominal responses are quite similar (Figure 14).

To quantify the effect of the resistance on the variability or robustness of the frequency response, it is possible to analyze the difference between the superior and inferior curves of the confidence interval. Smaller differences indicate smaller overall variability of the response for a given stochastic parameter and vice-versa. Although different metrics could be considered for such an analysis, here the mean differences between the superior and inferior curves over the frequency range of larger variability (2300 to $2700 \mathrm{~Hz}$ ) are used. For the smallest resistance considered, these reach up to around $3.7 \mathrm{~dB}$, for the independent circuit (Figure 13a), and around $4.0 \mathrm{~dB}$ (Figure 14a), for the interconnected circuit. On the other hand, these differences can be reduced to around $0.8 \mathrm{~dB}$ (Figure 13e) and $0.9 \mathrm{~dB}$ (Figure 14e), respectively, for the largest resistance considered.

As shown previously, global capacitance uncertainties lead to much larger frequency response variability. Hence, in these cases, the effect of increasing the resistance is even more important since it reduces this difference. Also, the mean difference between the superior and inferior values of the confidence interval can reach up to $10.8 \mathrm{~dB}$ (Figures $15 \mathrm{a}$ and 16a) for both circuits and the smallest resistance considered. This difference is reduced to around $2.7 \mathrm{~dB}$ for the largest resistance considered (Figures 15e and 16e).

These analyses can also be performed by combining both individual and global variability. This was done considering $5 \%$ relative dispersions for both $f_{i}$ and $f_{g}$. Figures 17 and 18 shows the nominal, mean and $95 \%$ confidence intervals for the FRF when considering five different values for the circuit resistance. It is noticeable that the results combine the features observed due to the separate dispersions. For the interconnected circuit and smallest resistance, the difference between the maximum of $95 \%$ confidence interval and the maximum nominal response is increased when considering both individual and global dispersions, compared to the 

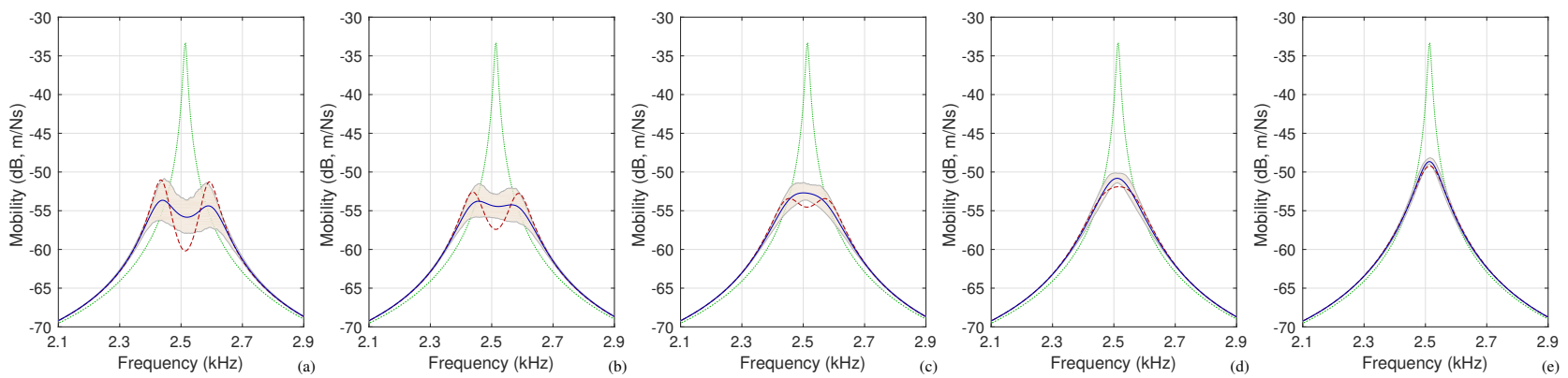

Figure 13. Frequency response of rod with independent circuits for uncertain patch capacitance (5\% individual dispersion) for decreased ((a): $-50 \%$, (b): $-30 \%)$, nominal (c) and increased ((d): $+40 \%,(e):+100 \%)$ resistances: open-circuit (short dash), RL nominal (dash), RL mean (solid), 95\% confidence interval (fill).
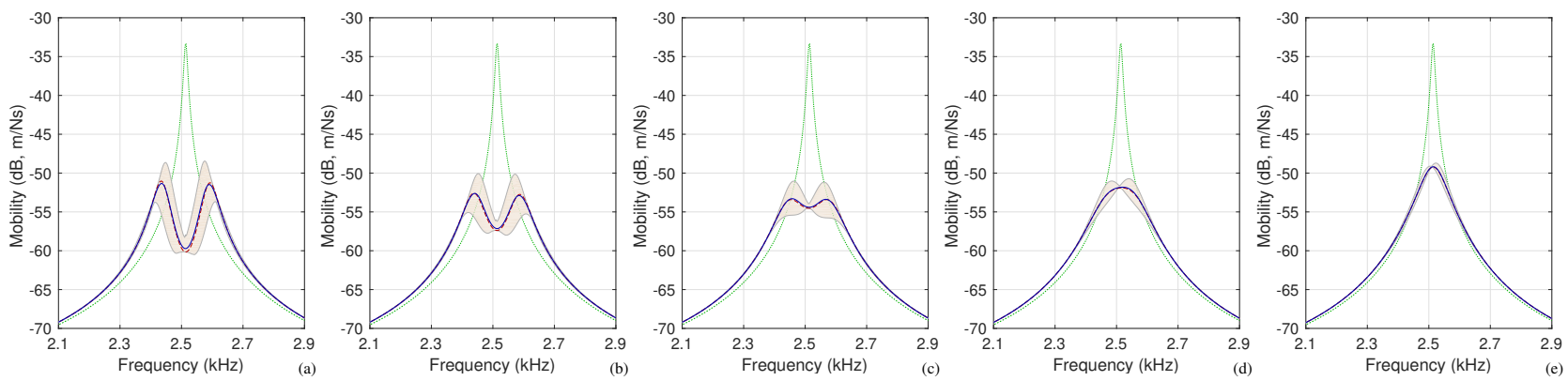

Figure 14. Frequency response of rod with interconnected circuits for uncertain patch capacitance ( $5 \%$ individual dispersion) for decreased ((a): $-50 \%$, (b): $-30 \%)$, nominal (c) and increased ((d): $+40 \%,(e):+100 \%)$ resistances: open-circuit (short dash), RL nominal (dash), RL mean (solid), 95\% confidence interval (fill).
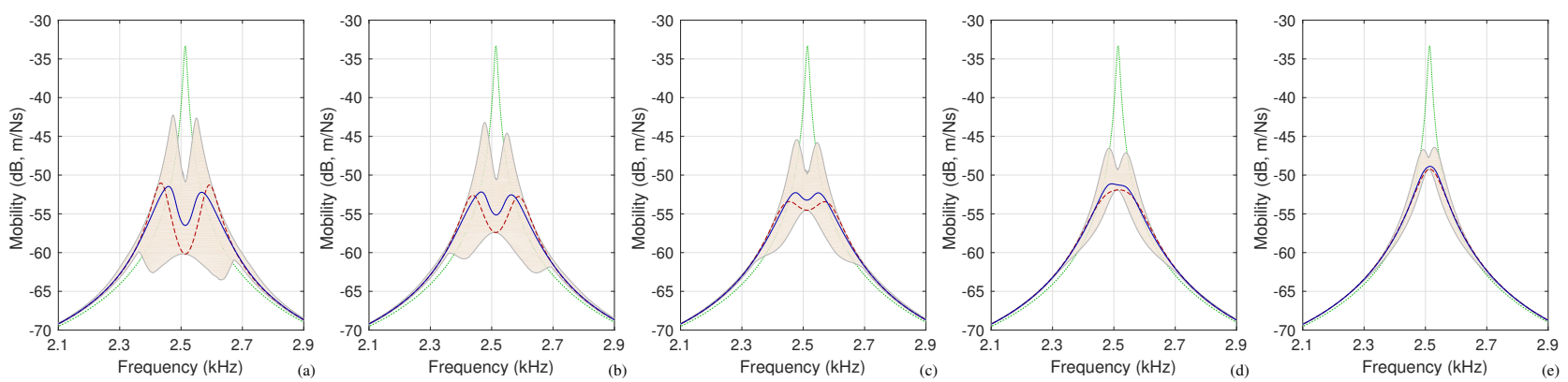

Figure 15. Frequency response of rod with independent circuits for uncertain patch capacitance (5\% global dispersion) for decreased ((a): $-50 \%$, (b): $-30 \%)$, nominal (c) and increased ((d): $+40 \%,(e):+100 \%)$ resistances: open-circuit (short dash), RL nominal (dash), RL mean (solid), 95\% confidence interval (fill).
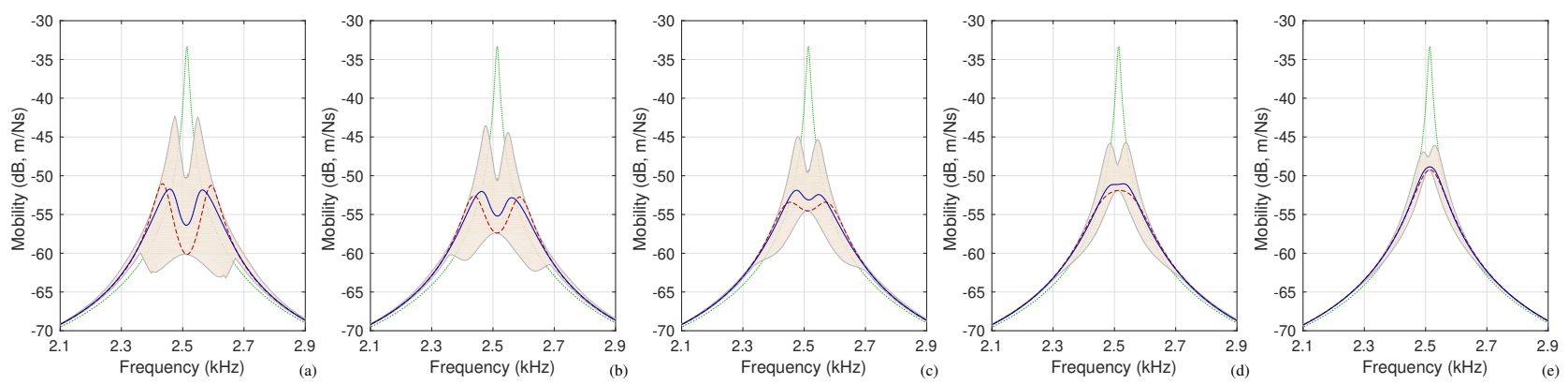

Figure 16. Frequency response of rod with interconnected circuits for uncertain patch capacitance (5\% global dispersion) for decreased ((a): $-50 \%$, (b): $-30 \%)$, nominal (c) and increased ((d): $+40 \%,(e):+100 \%)$ resistances: open-circuit (short dash), RL nominal (dash), RL mean (solid), 95\% confidence interval (fill).

case of global dispersion alone. On the other hand, for the independent circuit, this difference is actually decreased. However, for both circuits, the overall behavior indicates that increasing the resistance diminishes the variability, although not necessarily improving the robust performance. In the next section, two robust vibration mitigation performance criteria are proposed to provide a more direct way to quantify the effect of circuits' resistance for analysis and design for robustness. 

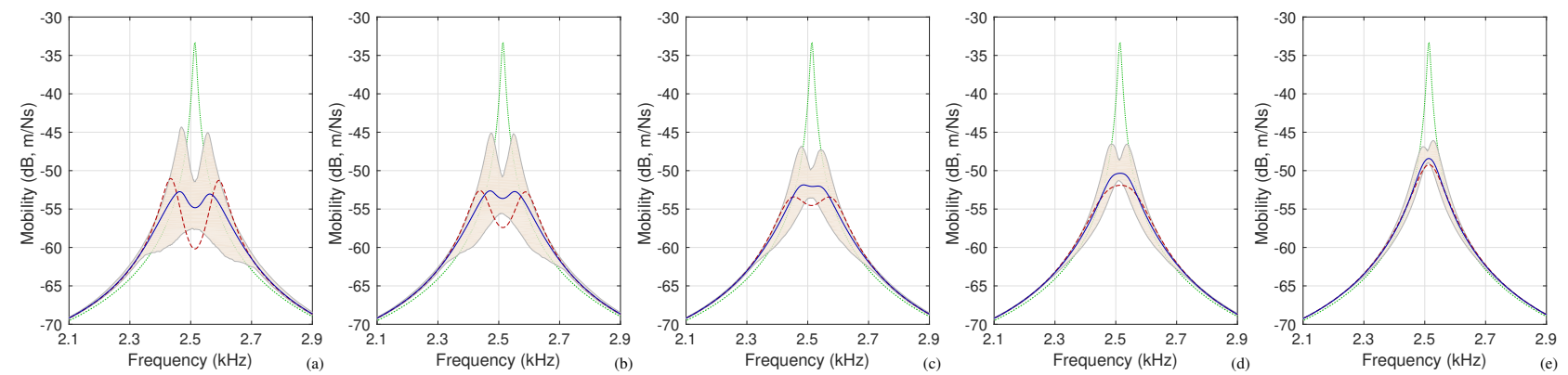

Figure 17. Frequency response of rod with independent circuits for uncertain patch capacitance $(5 \%$ individual dispersion and $5 \%$ global dispersion) for decreased ((a): $-50 \%$, (b): $-30 \%)$, nominal (c) and increased ((d): $+40 \%,(e):+100 \%)$ resistances: open-circuit (short dash), RL nominal (dash), RL mean (solid), 95\% confidence interval (fill).
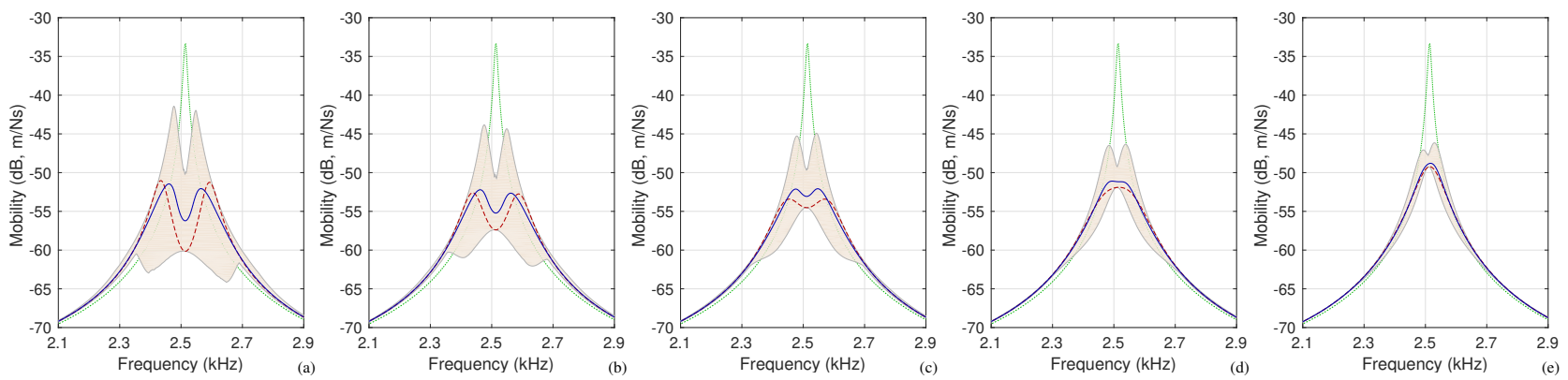

Figure 18. Frequency response of rod with interconnected circuits for uncertain patch capacitance (5\% individual dispersion and $5 \%$ global dispersion) for decreased ((a): $-50 \%$, (b): $-30 \%)$, nominal (c) and increased ((d): $+40 \%$, (e): $+100 \%)$ resistances: open-circuit (short dash), RL nominal (dash), RL mean (solid), 95\% confidence interval (fill).

\section{Effect of circuits' resistance on vibration mitigation performance}

Based on previous Monte Carlo simulation results, it was possible to conclude that, generally, increasing the circuits' resistance could lead to less variability in the frequency responses, which is in agreement with Andreaus and Porfiri (2007) and Berardengo et al. (2015). On the other hand, it is also worthwhile to analyze the effect of circuit resistance on the maximum response and, thus, on the potential vibration amplitude reduction under capacitance uncertainties. For that, the $H_{\infty}$ norms of each realization of the FRF responses were evaluated. Their distributions, nominal values and $95 \%$ confidence intervals were then assessed. The statistics of a second criteria, common to the design of resonant shunt circuits, based on the minimum damping factor is also analyzed. This was done by fitting the responses to a two degrees-of-freedom transfer function to extract the modal parameters from the FRFs.

From Figure 19a, it is possible to observe that for the independent circuit, from both FRF $H_{\infty}$ norm mean and maximum perspectives, the best resistance value (from those analyzed) is the one decreased by $30 \%$ from the nominal value. On the contrary, for the interconnected circuit, mean and maximum values of the FRF $H_{\infty}$ norm follow the behavior of its nominal value, as shown in Figure 20a. Thus, for the interconnected circuit, the nominal resistance value is also the best from both FRF norm mean and maximum perspectives. In terms of the minimum damping factor criteria, a resistance increased by around $60 \%$ is nominally the best solution. When considering the uncertainties, however, for the independent circuit, a resistance increased by $20 \%$ and $30 \%$ is the best choice from mean and minimum perspectives, respectively, although the variability diminishes for increasing resistance. For the interconnect circuit, the resistance increased by $60 \%$ is the best choice from nominal and mean perspectives, whereas the one increased by $50 \%$ should be the choice to maximize the minimum damping (Figures 19b and 20b).

This result has a strong consequence on practical design of physical inductors because, in order to satisfy the previous optima, the quality factor of the independent circuits has to be substantially larger than the quality factor of the interconnected circuits. Yet, in applications involving passive resonant shunts, the quality factor of a circuit is usually limited by the quality factor of the inductor itself, which already includes a non-negligible internal resistance (Lossouarn, Aucejo, Deü and Multon 2017). This does not promote the use of the independent circuits because designing inductors with a larger quality factor together with a larger inductance (see Table 1) would require larger coils and thus heavier electrical components.

On the other hand, in the case of capacitance global variability, both independent and interconnected circuits present the same overall behavior, in which larger resistance values are beneficial from both mean and maximum perspectives (Figures 21a and 22a). It is observed that the optimum in terms of mean value is obtained for a resistance increased by up to $40 \%$ from the nominal value for the independent case and by $20 \%$ for the interconnected case. In terms of minimum damping factor, the resistance increased by $60 \%$ is the best choice in all cases but the one to maximize the minimum interval for the independent circuit, in which case the performance can be marginally improved by further increasing the resistance (Figures $21 \mathrm{~b}$ and 22b). 


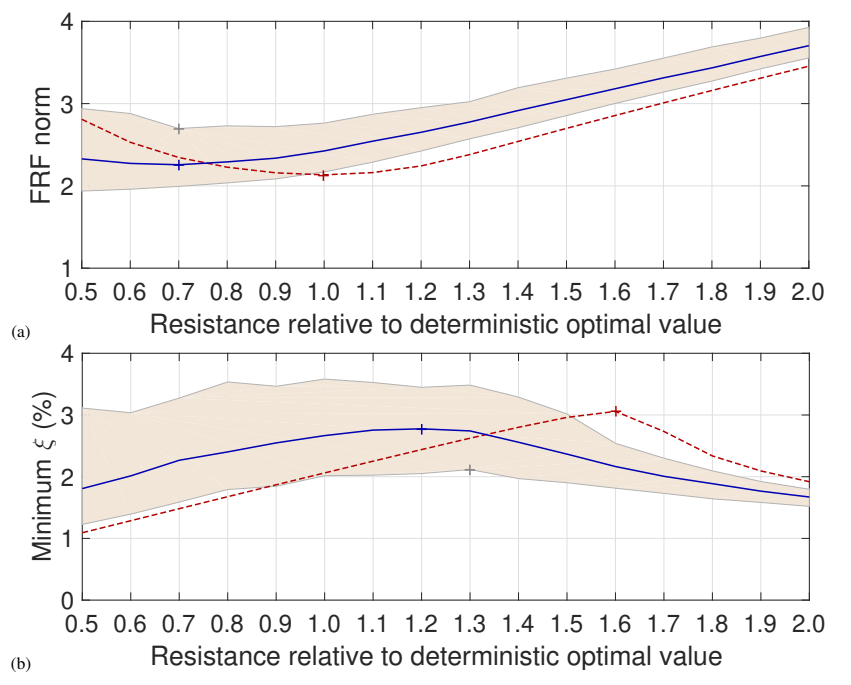

Figure 19. Statistics of the (a) norm of frequency response and (b) minimum damping of rod with independent circuits for uncertain patch capacitance ( $5 \%$ individual dispersion) with modified resistance: nominal (dash), mean (solid), 95\% confidence interval (fill).

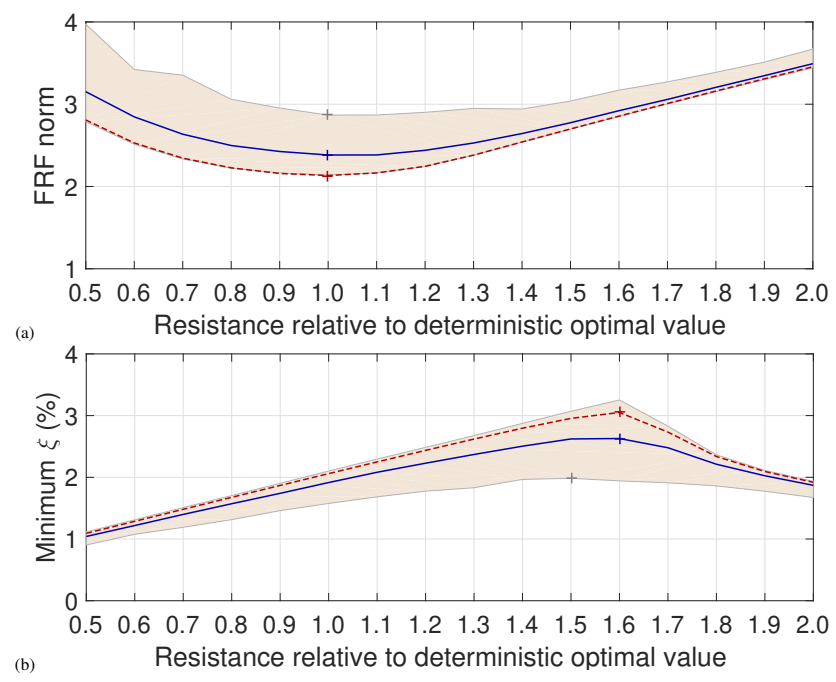

Figure 20. Statistics of the (a) norm of frequency response and (b) minimum damping of rod with interconnected circuits for uncertain patch capacitance ( $5 \%$ individual dispersion) with modified resistance: nominal (dash), mean (solid), 95\% confidence interval (fill).

These results are decisive because it is shown that the only circuit for which resistance values larger than the nominal one lead to better robust performances considering both individual and global variabilities is the interconnected one.

These analyses were also performed for combined individual and global variability. As shown in Figure 23a, since the robustness behaviors for individual and global variabilities are somewhat contradictory, the mean FRF norm is almost constant for the resistance values considered while the maximum FRF norm improves for larger resistance values. On the other hand, for the interconnected circuit, $10 \%$ and $40 \%$ increased resistance values improve robustness using mean and maximum criteria, respectively (Figure 24a). In terms of minimum damping factor, for the independent circuit, resistance values closer to the nominal one provide higher minimum damping from the mean
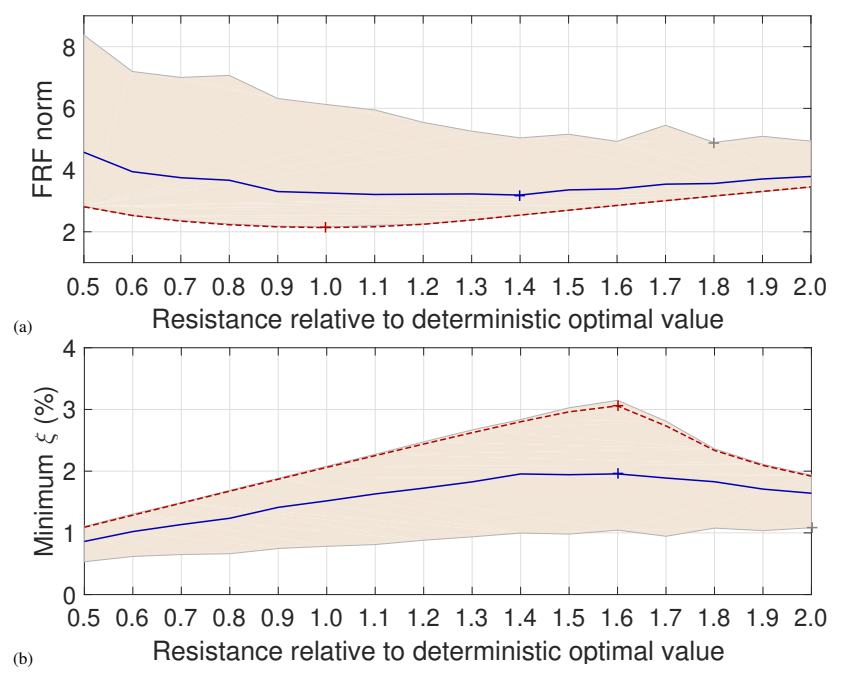

Figure 21. Statistics of the (a) norm of frequency response and (b) minimum damping of rod with independent circuits for uncertain patch capacitance ( $5 \%$ global dispersion) with modified resistance: nominal (dash), mean (solid), 95\% confidence interval (fill).

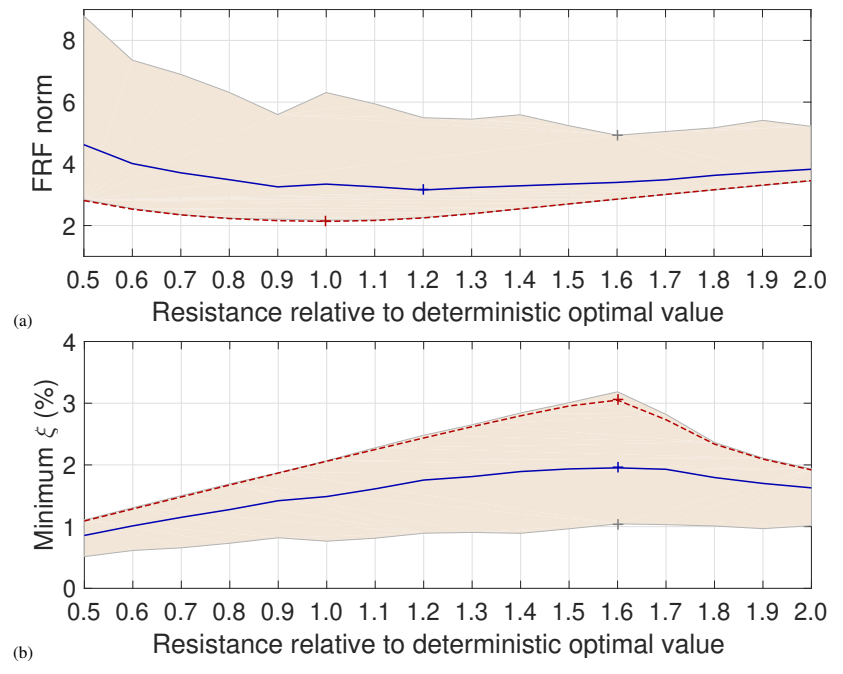

Figure 22. Statistics of the (a) norm of frequency response and (b) minimum damping of rod with interconnected circuits for uncertain patch capacitance ( $5 \%$ global dispersion) with modified resistance: nominal (dash), mean (solid), 95\% confidence interval (fill).

perspective, although higher resistances marginally improve the minimum interval (Figure 23b). For the interconnected circuit, from nominal, mean and maximum perspectives, resistances increased by $60 \%, 50 \%$ and $100 \%$ are the best choices, respectively (Figure 24b).

\section{Effect of uncertainties on multimodal vibration mitigation}

While the interest of interconnected circuits has been proven for single-mode vibration mitigation, one can remember that the main advantage of those circuits is to provide damping on several modes simultaneously. A perspective of the present work is thus to investigate the effect of uncertainties on multimodal vibration mitigation. 


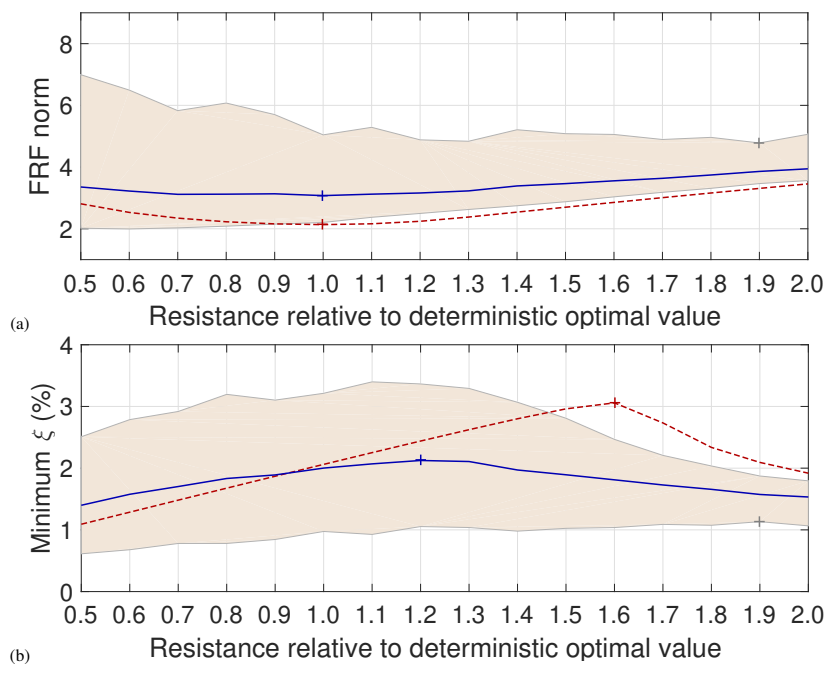

Figure 23. Statistics of the (a) norm of frequency response and (b) minimum damping of rod with independent circuits for uncertain patch capacitance (5\% individual dispersion and $5 \%$ global dispersion) with modified resistance: nominal (dash), mean (solid), 95\% confidence interval (fill).

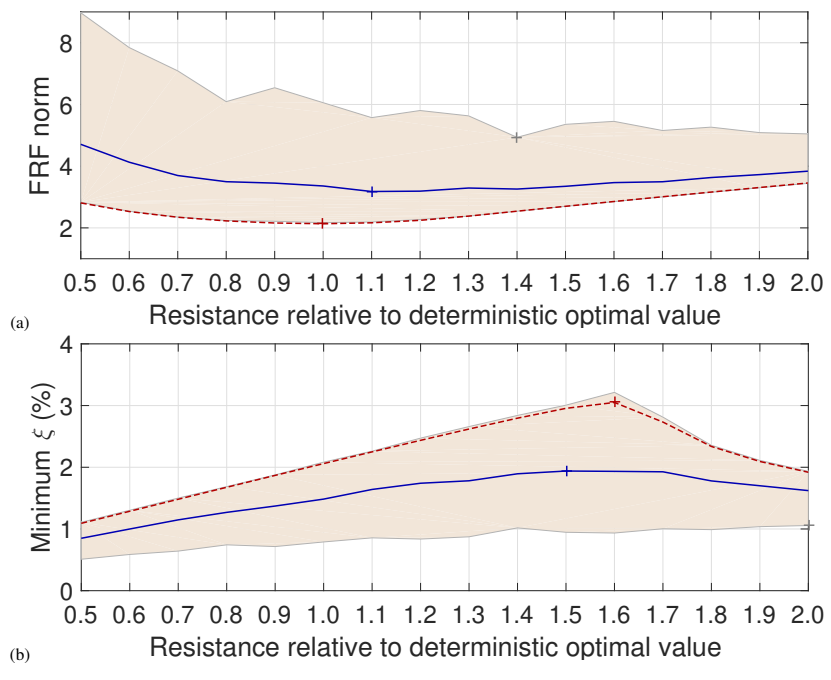

Figure 24. Statistics of the (a) norm of frequency response and (b) minimum damping of rod with interconnected circuits for uncertain patch capacitance (5\% individual dispersion and $5 \%$ global dispersion) with modified resistance: nominal (dash), mean (solid), 95\% confidence interval (fill).

Preliminary results, as shown in Figure 25, indicate that the vibration mitigation performance for all modes is affected by the capacitance uncertainties. It is first noticed the increasing detuning on the nominal and mean FRF curves where the pairs of local maxima do not have the exact same amplitude. This effect is actually not related to uncertainties but to the number of unit cells in the electrical network (Lossouarn, Aucejo and Deü 2015). Increasing the number of components would better tune simultaneously both mean and nominal responses.

Yet, the difference between the nominal response and the mean or maximum responses strongly increases for the highest modes. This is mainly due to the lower damping of the highest modes. Indeed, damping is introduced with a resistance in series with the inductance, as seen in Figure 1. Impedance calculation shows that the resulting quality factor $L_{s} \omega / R_{s}$ increases with the frequency leading to underdamped highest mode when the first mode is correctly tuned. Lower damping then amplifies the effect of capacitance variabilities, as seen from Figure 18c to Figure 18 .

While broadband tuning of damping coefficients is out of the scope of the present paper, those results highlight the need of an optimized damping thanks to adequate resistors placed at different positions of the electrical network. This makes a direct perspective of the present work in order to propose an interconnected network for both multimodal and robust vibration mitigation.

\section{Conclusions}

This work has presented an analysis of the effect of parametric uncertainties on the vibration control performance of a rod with periodically distributed piezoelectric patches that are either independently connected to electrical shunt circuits or interconnected through an electrical line of inductors. In both cases, the capacitances of piezoelectric patches were considered as stochastic parameters following a known probability density function distribution. Then, Monte Carlo simulations were performed to evaluate mean values and confidence intervals of the frequency response functions to assess the robustness of each solution and to compare different solutions in terms of nominal and robust performances. Results have shown that vibration amplitude reduction worsen significantly (with loss in reduction of up to $13 \mathrm{~dB}$ in the proposed example) due to the mistuning between structural natural frequency and circuit resonance frequency. Yet, interconnected circuits are more robust to individual capacitance variabilities than independent shunt ones because they are able to maintain a mean response that is very close to the nominal one.

Considering the clearly different responses between independent and interconnected circuits subjected to individual variabilities, it was proposed to assess the effect of modifying the circuits' resistance on the vibration reduction performance robustness. Results have shown that, for the interconnected case, the optimal resistance for robust performance is the same as the nominal one. On the other hand, for independent shunt circuits and individual variabilities, decreasing the resistance has shown to be a better compromise solution. This induces a practical limitation for those independent shunts because they require both larger inductance and quality factors that could lead to bulky physical component when considering a passive realization.

Another issue concerns the robust optimum when considering global variation of the circuits' capacitance. In this case, the resistance always has to be larger than its deterministic optimal one, which is in contradiction with the previous results. In the end, even without considering its broadband capabilities, all of this make the interconnected circuit the best candidate for a robust design of a resonant piezoelectric array intended for vibration mitigation. Future works will be directed to the extension of this approach to assess the effect of parametric uncertainties on multimodal vibration mitigation. 


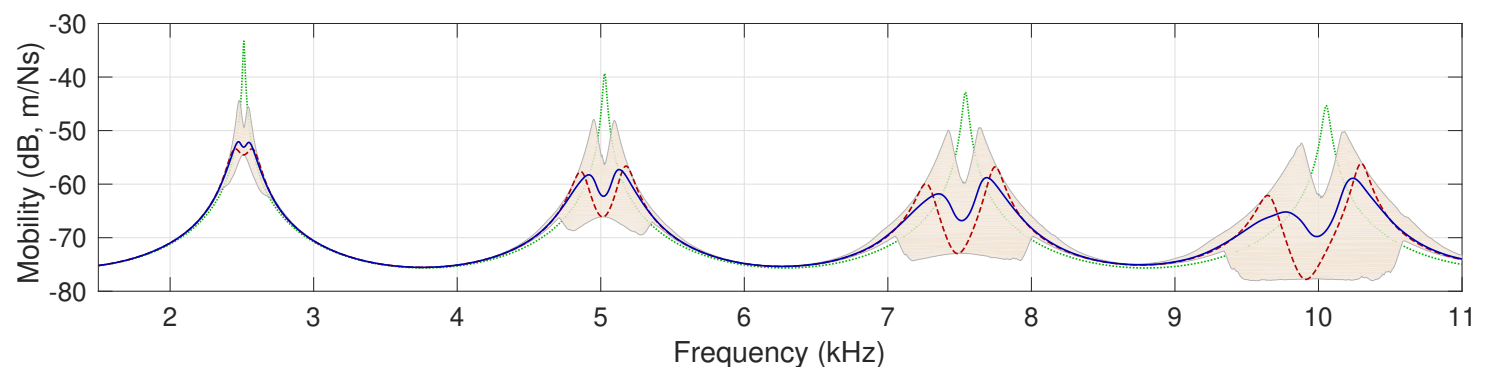

Figure 25. Wideband frequency response of rod with interconnected circuits for uncertain patch capacitance considering $5 \%$ individual and global dispersions: open-circuit (short dash), RL nominal (dash), RL mean (solid), 95\% confidence interval (fill).

\section{Acknowledgements}

Financial support of Coordination for the Improvement of Higher Education Personnel (CAPES), CAPES/Cofecub grant 913/18, and National Council for Scientific and Technological Development (CNPq), grants 309193/2014-1 and 309001/2018-8, is gratefully acknowledged.

\section{References}

Ahmadian M and DeGuilio AP (2001) Recent advances in the use of piezoceramics for vibration suppression. Shock and Vibration Digest 33(1): 15-22.

Airoldi L and Ruzzene M (2011) Design of tunable acoustic metamaterials through periodic arrays of resonant shunted piezos. New Journal of Physics 13: 113010.

Andreaus U and Porfiri M (2007) Effect of electrical uncertainties on resonant piezoelectric shunting. Journal of Intelligent Material Systems and Structures 18: 477-485.

Berardengo M, Cigada A, Manzoni S and Vanali M (2015) Vibration control by means of piezoelectric actuators shunted with LR impedances: performance and robustness analysis. Shock and Vibration 2015: 704265.

Bergamini A E, Zündel M, Flores Parra E A, Delpero T, Ruzzene M and Ermanni P (2015) Hybrid dispersive media with controllable wave propagation: A new take on smart materials. Journal of Applied Physics 118(15): 154310.

Flores Parra E A, Bergamini A, Lossouarn B, Van Damme B, Cenedese M and Ermanni P (2017) Bandgap control with local and interconnected LC piezoelectric shunts. Applied Physics Letters 111(11): 111902.

Celli P and Gonella S (2015) Tunable directivity in metamaterials with reconfigurable cell symmetry. Applied Physics Letters 106: 091905.

Darleux R, Lossouarn B and Deü J-F (2018) Passive selftuning inductor for piezoelectric shunt damping considering temperature variations. Journal of Sound and Vibration 432: 105-118.

Hagood NW and von Flotow A (1991) Damping of structural vibrations with piezoelectric materials and passive electrical networks. Journal of Sound and Vibration 146(2): 243-268.

Hollkamp JJ (1994) Multimodal passive vibration suppression with piezoelectric materials and resonant shunts. Journal of Intelligent Materials Systems and Structures 5(1): 49-57.

Jaynes ET (1957) Information theory and statistical mechanics. Physical Review 106(4): 620-630.

Lossouarn B, Aucejo M and Deü J-F (2015) Multimodal coupling of periodic lattices and application to rod vibration damping with a piezoelectric network. Smart Materials and Structures 24: 045018 .

Lossouarn B, Aucejo M, Deü J-F and Multon B (2017) Design of inductors with high inductance values for resonant piezoelectric damping. Sensors and Actuators A: Physical 259: 68-76.

Maurini C, Dell'Isola F and Del Vescovo D (2004) Comparison of piezoelectronic networks acting as distributed vibration absorbers. Mechanical Systems and Signal Processing 18: 1243-1271.

Preumont A (2018) Vibration control of active structures: an introduction (Vol. 246). Springer.

Reza Moheimani SO (2003) A survey of recent innovations in vibration damping and control using shunted piezoelectric transducers. IEEE Transactions on Control Systems Technology 11(4): 482-494.

Santos HFL and Trindade MA (2011) Structural vibration control using extension and shear active-passive piezoelectric networks including sensitivity to electrical uncertainties. Journal of the Brazilian Society of Mechanical Sciences and Engineering 33: 287-301.

Soize C (2001) Maximum entropy approach for modeling random uncertainties in transient elastodynamics. Journal of the Acoustical Society of America 109(5): 1979-1996.

Thomas O, Ducarne J and Deü J-F (2012) Performance of piezoelectric shunts for vibration reduction. Smart Materials and Structures 21(1): 015008.

Thorp O, Ruzzene M and Baz A (2001) Attenuation and localization of wave propagation in rods with periodic shunted piezoelectric patches. Smart Materials and Structures 10: 979989.

Thorp O, Ruzzene M and Baz A (2005) Attenuation of wave propagation in fluid-loaded shells with periodic shunted piezoelectric rings. Smart Materials and Structures 14: 594604.

Valis T, von Flotow AH and Hagood NW (1994) An acousticelectromagnetic piezoelectric waveguide coupler. Journal of Sound and Vibration 178: 669-680. 